\title{
PATCHES FOR ACNE TREATMENT: AN UPDATE ON THE FORMULATION AND STABILITY TEST
}

\author{
TAQIYYAH QOTHRUNNADAA*, ALIYA NUR HASANAH
}

Faculty of Pharmacy, Universitas Padjadjaran, Jl. Raya Bandung Sumedang KM 21.5, Sumedang 45363, Indonesia

Email: aliya.n.hasanah@unpad.ac.id

Received: 06 Aug 2021, Revised and Accepted: 17 Oct 2021

\begin{abstract}
The purpose of this article review was to find out more about acne patch formulations and test stability. The method used is searching data from various articles based on Google Scholar, Elsevier, and Molecules with the keywords "Acne vulgaris, acne treatment, acne patches, and types of acne patches" and with a range of years between 2011 and 2021. Based on the type of acne, acne patches are divided into several types, namely microneedle patches, acne medicine patches, and hydrocolloid patches. The results obtained in this review show that the most commonly used acne patch is the medicated acne patch (hydrogel patches), because of its waterproof property to protect acne from secondary infection, it can absorb liquid inside and flatten acne and is cheaper compared to microneedles. The hydrogel patch is also stable at a temperature of $40 \pm 2{ }^{\circ} \mathrm{C}$ and a relative humidity (RH) of $75 \pm 5 \%$ for 6 mo of storage.
\end{abstract}

Keywords: Acne vulgaris, Acne patches, Microneedle patches, Hydrogel patches, Hydrocolloid patches, Stability

(C) 2021 The Authors. Published by Innovare Academic Sciences Pvt Ltd. This is anopen access article under the CC BYlicense (https://creativecommons.org/licenses/by/4.0/) DOI: https://dx.doi.org/10.22159/ijap.2021.v13s4.43812 Journal homepage: https://innovareacademics.in/journals/index.php/ijap

\section{INTRODUCTION}

Acne vulgaris is an inflammatory disease that occurs in the sebaceous unit of the hair follicle. It is usually chronic and heals on its own. Acne vulgaris is caused by Cutibacterium acnes in adolescence, under the influence of normal circulating dehydroepiandrosterone (DHEA). Acne vulgaris is a very common skin disorder and can cause inflammatory and non-inflammatory lesions, especially on the face. However, it can also occur on the arms, body, and back. Globally, the prevalence of acne vulgaris ranks 8th among all diseases, with the highest order being in Western Europe. The urban population is higher than in rural areas. About $20 \%$ of individuals who get severe acne will cause scarring $[1,2]$

Chronic inflammation that occurs in acne can include open comedones (blackheads), closed comedones (whiteheads), and inflammatory lesions such as papules, postules, and nodules. Acne is considered as a chronic disease because it can affect the sufferer psychologically. There are several negative effects due to acne on adolescents, including emotional stress, discomfort, damage to the skin, and can even cause permanent scarring on the skin. These things can create anxiety and embarrassment in the sufferer [3].

Increased production and severity of acne can be influenced by several factors, namely, male gender, stress, smoking, youth, and comedogenic drugs such as halogens, androgens, corticosteroids, and cosmetics that can clog pores. Based on previous research, it is known that genetic influences such as in men when combined with comedogenic hormones (especially androgens) will cause abnormal sebum volume and contribute to acne lesions [3].

Acne is classified into several forms, namely (1) Comedo (whiteheads or blackheads) or papules which are basic acne lesions or clogged pores. Whiteheads are white bumps because the clogged pores stay under the skin or are called closed comedones. While blackheads are open comedones with a blackish color on the skin surface. But the black color is not caused by dirt but because the air reacts with excess oil. (2) Pustules (pimples) are white or yellowish pus-filled lesions that are red at the base. (3) Nodules are acne lesions that are more serious because they stick to the deep skin, are painful, and cause scarring. And (4) Cyst are like a nodule; the lesion is deep in the skin, painful, pus-filled lesions that can cause scarring [4].

Based on the American Academy of Dermatology Association (2016), treatment management for acne is classified based on its severity [5]. When assessing the severity of acne, there are several things to consider, such as the distribution (back, upper arms, chest), type and number of lesions (nodules, blackheads, papular pustules), and the presence or absence of scarring (table 1) [6].

Table 1: Grading severity of acne

\begin{tabular}{lll}
\hline Grade & Severity & Clinical findings \\
\hline I & Mild & Open and closed acne, rarely inflammatory papules and pustules \\
II & Moderate & Papules and pustules, mainly on face \\
III & Moderately severe & There are a large number of papules and pustules on the chest and back, and \\
& occasionally inflamed nodules. \\
IV & Severe & Many large, painful nodules and pustules \\
\hline
\end{tabular}

Acne is commonly affecting many teenagers that makes so many scientists are exploring many formulas for acne treatment. There is a new invention for topical acne treatment called acne patch. However, until now, the article that discusses acne patches has never been made. Therefore, this article is written to describe acne treatment with acne patch. This article contains treatments for acne, especially acne patches as one of the current acne treatments. It also explains the types and formulations of acne patches and acne patch stability test.

\section{Acne treatment}

There are several common forms of treatment for acne, such as topical therapies, systemic medication, physical therapy, laser, and photodynamic therapy [7]. Topical therapy is standard therapy for mild to moderate acne. Mainstays of topical acne therapy are retinoids and antibacterial such as benzoyl peroxide and antibiotics. Topical therapy can prevent new lesions but can cause local irritation. The side effect of gels, pledges (medication-soaked pads), washes and solutions is to dry out the skin so it tends to be used for oily skin. Meanwhile, lotions, creams, and ointments can be used for dry skin, but the risk of irritating the skin is higher.

This topical therapy takes at least six to eight weeks for results to be seen [6]. The differences between each preparation in the use of topical acne treatment are described in table 2 [8]. 
Table 2: Acne treatment dosage forms and their functions

\begin{tabular}{ll}
\hline Preparation & Function \\
\hline Gel & $\begin{array}{l}\text { Gel is used for patients with oily skin, because it is more comfortable and has a drying effect. However, it can cause } \\
\text { burning-type irritation and prevent certain types of cosmetics from adhering to the skin } \\
\text { Its main solution is used with topical antibiotics dissolved in alcohol. Just like the gel, the solution is also used for } \\
\text { patients with oily skin } \\
\text { Lotions can be used on all skin types. The lotion spreads well all over the hair-bearing skin. However, usually lotions } \\
\text { contain propylene glycol, causing a burning and drying effect. } \\
\text { Cream is used for patients with dry or sensitive skin, so it requires a formulation that does not dry out the skin and } \\
\text { does not cause irritation to the skin. If it is given to patients with oily skin it will feel very oily with the use of cream. }\end{array}$ \\
\end{tabular}

The goals of treatment in patients with acne are preventing discharge scars, reduction of psychological morbidity, and resolution of inflammatory and non-inflammatory lesions. To assess the treatment is going well or not, therapy should be given for at least eight-month. If the goal of treatment is not achieved or there is significant scarring, a referral to a dermatologist is recommended [9].

Many antibacterial agents for the treatment of acne have been explored, such as erythromycin, clindamycin (CDM) and benzoyl peroxide, but not all routes of administration can be effective and safe against acne. Most of these drugs do not kill bacteria effectively because of poor penetration into the dermis. Topical antibiotics are not considered single therapy for acne because they pose a risk of bacterial resistance. Meanwhile, oral antibiotics or isotretinoin showed better therapeutic efficacy [10]. Oral antibiotics are given to patients with moderate to severe acne severity and in patients with inflammatory acne that is resistant to topical treatments although oral antibiotics show better therapeutic efficacy, their use should be limited because they can cause inflammatory bowel disease, pharyngitis, induction of Candida vulvoganitis, and Clostridium difficile infection. The recommended duration of antibiotic treatment is 6 to $8 \mathrm{w}$ with a maximum of 12 to $18 \mathrm{w}$. repeated antibiotic treatment is recommended using the same antibiotics and using benzoyl peroxide for at least 5 to $7 \mathrm{~d}$ to reduce resistant organism. The number of side effects that arise in the use of topical or oral therapy makes most consumers want to find other formulations with better therapeutic efficacy and side effects can be desired $[7,10,11]$.

Early use of topical therapy, patches were most used as the transdermal delivery system, such as scopolamine patch for the treatment of motion sickness, fentanyl patch for the treatment of pain, and nicotine patch for smoking reduction and cessation aid. The purpose of this patches is to avoid the first-pass metabolism and transdermal blood level distribution. Over time, drug candidates suitable for transdermal delivery have been defined, for example, for cosmetics needs. There is a focus for finding drugs that contains sufficiently potent drugs that can penetrate into the skin with transdermal technology, such for healing acne [12]. Recently, there is a popular acne treatment, namely acne patches. Acne patches are in great demand because they have the ability to conceal and treat acne at the same time. Acne patches are also easier to buy, to use, and easier to carry on, so they are very popular among consumers.

\section{Patches as a topical acne treatment}

In general, acne patches that are already on the market contain hydrocolloid synthesizing substances [13]. Acne patches on the market are usually made of hydrocolloids or hydrogels; these substances are known for their use in medical dressings. Hydrocolloid dressings consist of two layers, namely, a colloid layer and a waterimpermeable layer. The so-called colloid layer is the inner layer and the water-impermeable layer is the outer layer. The function of the water-impermeable layer is that it can provide a protective layer and can help prevent the spread of pathogenic microorganisms, but this water-impermeable layer can create hypoxic conditions and increase the growth of P. acnes. This water-impermeable coating is not efficient at treating acne due to its protective function, so some medical-grade patches are required containing several substances such as benzoyl peroxide, salicylic acid, or chlorhexidine which can inhibit bacterial growth [14]. Based on the type of acne, acne patches are divided into several types (table 3).

Table 3: Types of acne patches [15]

\begin{tabular}{ll}
\hline Acne patches & Characteristics \\
\hline Microneedle patches & - For cystic or nodular acne \\
& - Containing microneedle that is dissolved and is very fine, has a small needle on one side \\
Medicated acne & - Can help deliver and penetrate the active ingredients into the deeper layers of the skin \\
patches (Hydrogel & - Contains active ingredients to kill acne-causing bacteria and reduce inflammation. \\
patches) & - The active ingredients commonly used in this type of acne patch are salicylic acid and tea tree oil. \\
& - Can reduce bumps, pain, and redness in pimples, and can relieve inflamed pimples such as papules. \\
Hydrocolloid patches & - For yellow or white pimples. \\
(non-medicated acne & - Often used to speed up the healing process. \\
patches) & - Has the size of a pimple and is circular in shape \\
& - The shape is also very thin so that when used in public it is less visible. \\
& - Can suck moisture from the pores, prevent other infections, and can prevent the formation of acne scars on the skin.
\end{tabular}

\section{Microneedle (MN) patches}

Microneedle (MNs) patches is a patch with a topical, transdermal drug delivery system with a material made of micrometer-sized needles. This system with a micrometer needle arrangement creates a transportation route through the skin to transport various therapeutic molecules, such as (bio) macromolecules, small molecules, and nanoparticles (vesicles). These microneedle patches are adapted so that they can only penetrate the epidermis without penetrating the dermis and do not damage the neurons. As this patches is usually administered at home, these patches were designed to be pain free and easy to use [16].

To prepare microneedle patches, various materials such as silicones, metals, polymers and ceramics have been used. However, in one study, materials that could increase transdermal drugs were biodegradable or dissolvable, such as silk fibroin or hyaluronic acid, and nondegradable polymer micronedle with (or without) cross-linked structures, such as polyvinyl alcohol or methacrylated hyaluronic acid [16]. The differences of each material are described in table 4. 
Table 4: Advantages and disadvantages of materials used in microneedle patches

\begin{tabular}{|c|c|c|}
\hline Material & Advantages & Disadvantages \\
\hline Silicones & $\begin{array}{l}\text { Silicon microneedles has excellent biocompatibility, particularly in mechanical } \\
\text { properties superior to those of polymer and metal, such as a non-ductility and } \\
\text { indentation hardness, and can penetrate the skin without causing any damage to the } \\
\text { skin [11]. }\end{array}$ & $\begin{array}{l}\text { Silicon materials have high cost, intricate } \\
\text { fabrication requirements, long fabrication } \\
\text { times, and complex, and risk of practicable } \\
\text { fracture on the skin [17]. }\end{array}$ \\
\hline Metals & $\begin{array}{l}\text { Metal microneedles have sufficient mechanical strength to penetrate the skin among } \\
\text { the others materials [18]. Most common metals used are stainless stell, titanium, } \\
\text { platinum, alloys, and gold [17]. }\end{array}$ & $\begin{array}{l}\text { Metals have a high potential biological } \\
\text { waste and expensive cost [18]. }\end{array}$ \\
\hline Polymers & $\begin{array}{l}\text { Polymers microneedles has overcome the limitations of silicon microneedles and } \\
\text { metal microneedles, and can provide low cost, mechanical strength and safety to } \\
\text { prevent the needle from accidentally breaking in the skin [19]. Most common used } \\
\text { polymers are CMC, polyvinyl alcohol, PLGA, PVP [17]. }\end{array}$ & $\begin{array}{l}\text { Polymers have less strength than metals to } \\
\text { penetrate into the skin [19]. }\end{array}$ \\
\hline Ceramics & $\begin{array}{l}\text { Higher mechanical strength and better stability at high temperature and humidity } \\
\text { than polymers. Also, they provide controllable porosity and easy handling and } \\
\text { adjustable porosity during the production process, so ceramic surfaces and penetrants } \\
\text { can be used to enhance the penetration of transdermal delivery [20]. Most common } \\
\text { used ceramics are alumina, calcium phosphate, and calcium sulphate [17]. }\end{array}$ & $\begin{array}{l}\text { Usually sintered under high temperature } \\
\text { and makes it challenging to load } \\
\text { thermolabile medications into the ceramic } \\
\text { material [20]. }\end{array}$ \\
\hline
\end{tabular}

The advantages of microneedles itself are (1) many macromolecules can be administered (2) the active pharmaceutical ingredient is applied painlessly on the skin (3) not affected by the first-pass metabolism (4) easy administration and rapid drug delivery, which can be targeted on the specific area, and (5) good tolerability without causing edema or erythema on the skin. Meanwhile, the disadvantages are (1) the risk of "bouncing off" on the skin surface (2) external environment such as hydration on the skin could affect the drug delivery (3) repetitive injections may collapse the veins (4) the difference of skin layers one people to another may cause the penetration depth of particles could be vary (5) the tip of the microneedle may break out and remain within the skin on removal of the patch [19].

Microneedle contains drugs that enter the skin to form microchannels in the epidermis. These micro-channels are smaller than a syringe hole but larger in molecular dimensions. The micro-channels that are formed can be the entry points for bacteria and cause infection; this is the reason the application of antimicrobial microneedles are to inhibit bacterial infection [16].

In a study by Zhang et al. 2020 Microneedle patches were made from poly (ionic liquids) (PILs) as a base material, which is a group of polymers derived from ionic liquid monomers (IL) and has high physical and chemical stability. The cationic functional groups of PIL, namely imidazolium, pyrrolidinium, and quaternary ammonium indicate that PIL is intrinsically antibacterial [16]. In that study, tests were performed by combining PIL and microneedles to provide an easy and effective strategy for the treatment of acne infections. This patch contains the salicylic acid anion in PIL-MN, and through the electrostatic interaction between salicylic acid anion and imidazolium cation, it is ensure that part of the salicylic acid can enter the skin surface with micro channels on the pain-free microneedle. The prepared PIL-MN patch shows high mechanical strength and antibacterial properties against microorganisms such as Escherichia coli (E. coli), Staphylococcus aureus (S. aureus), and Propionibacterium acnes [16].
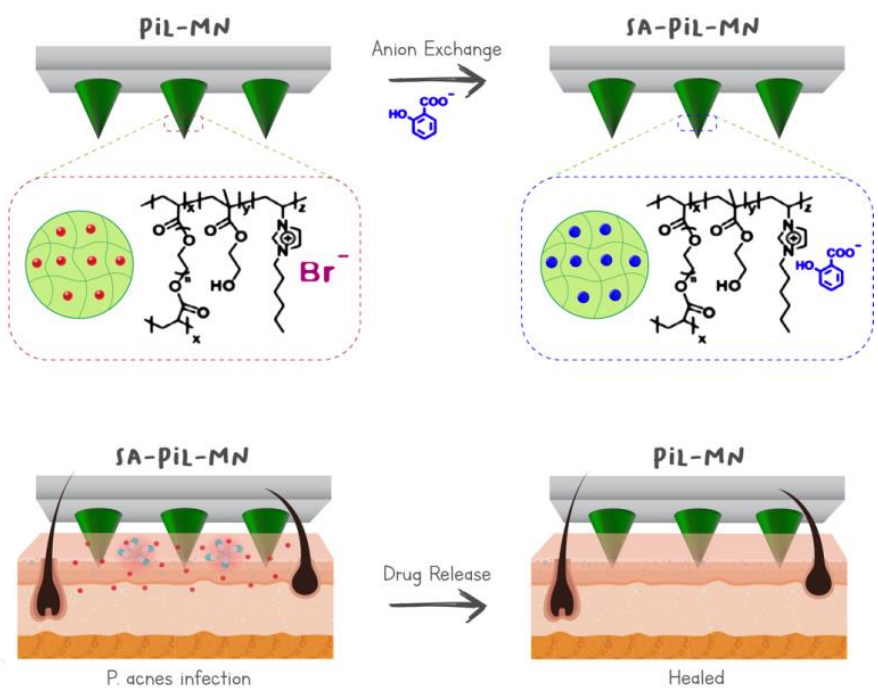

Fig. 1: Illustration of SA-PIL-MNs preparation and SA-PIL-MNs release during treatment of P. acnes infection

Microneedle patch can accelerate the transdermal efficiency of salicylic acid and enhance the therapeutic effect on infection. Tests were carried out on mice with various treatments. SA-PIL-MNs proved to be the most effective in treating acne infections on the skin of mice [16].

Based in vivo study by Zhang et al. 2020 the test Antibacterial activity of PIL-base MNs shows that SA solution can effectively inhibit the growth of P. acnes, with an antibacterial rate of about
$89.37 \%$, which is much higher than $66.88 \%$ of PIL-MN. However, once SA- anions are introduced into the PIL-MN system, the bacterial viability of P. acnes will be significantly reduced (almost zero). These results show that the use of SA and PIL-MN alone has a moderate antibacterial effect and that SA-PIL-MN has the best effect in killing P. acnes in combination with SA and PIL-MN [16]. Microneedles can generally be classified into four types according to drug delivery methods. There are several types of microneedles' mechanism that were described in table 5 [21]. 
Table 5: Types of microneedle

\begin{tabular}{ll}
\hline Classification & Formulation \\
\hline Solid Microneedle & $\begin{array}{l}\text { The solid silicon long tapered microneedles using tetramethylammonium hydroxide have an average height of 158 } \mu \text { m } \\
\text { and a base width of } 110.5 \mu \mathrm{m} . \text { In order to enhance drug absorption, the microneedles have a depth of } 800 \mu \mathrm{m} \text { and a } \\
\text { density of } 256 \mathrm{MNs} \text { per } \mathrm{cm}^{2} .\end{array}$ \\
Coated Microneedle & $\begin{array}{l}\text { This kind of microneedles is sorrounded by a drug solution or a drug dispersion layer, so the amount of drug depends on } \\
\text { the thickness of the coating, such as PLA microneedles with sulforhodamine B has an efficiency was approximately } 90 \% .\end{array}$ \\
Dissolving Microneedle & $\begin{array}{l}\text { By encapsulating drugs in polymer, biodegradable polymers are used to make soluble MN. The polymer-drug mixing gave } \\
\text { sufficient mechanical strength, approximately } 90 \% .\end{array}$ \\
Hollow Microneedle & $\begin{array}{l}\text { Hollow microneedles have holes at the tips that will be filled with the drug dispersion. To develop hallow MN, a metal } \\
\text { coat or fused silica is applied to increase the strength of the microneedle. }\end{array}$ \\
\hline
\end{tabular}

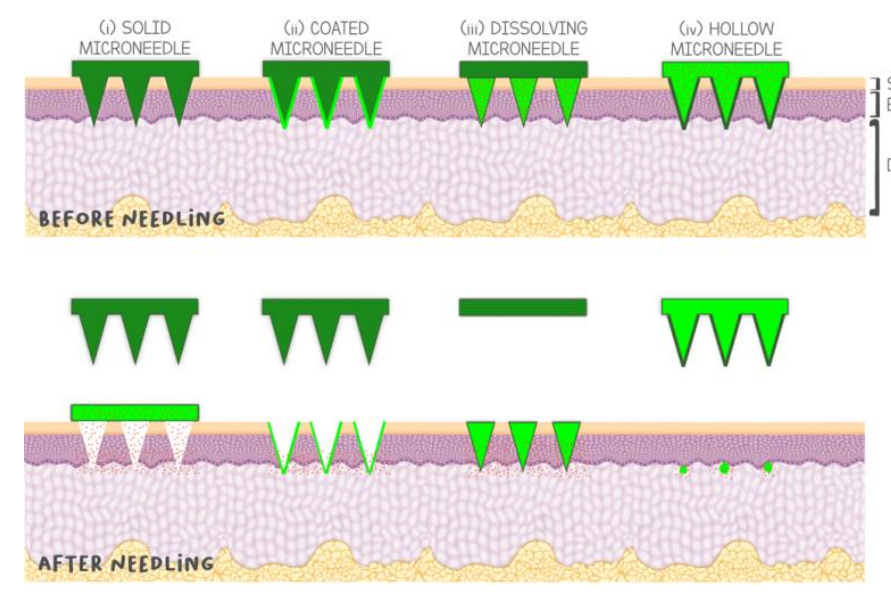

Fig. 2: Illustration of different types of microneedles on the skin

\section{Hydrogel patches}

Medicated acne patches are also called hydrogel patches because hydrogel patches contain active ingredients that can kill acnecausing bacteria. Most of the anti-acne patches are hydrocolloid or hydrogel stickers which are waterproof on the surface to protect acne from secondary infection. The hydrocolloid patch can absorb fluid on the pimples and make the pimples flatten [14]. Hydrogel is a cross-linked hydrophilic polymer network with a high water content. The high water content of the hydrogel makes biocompatibility beneficial, so the hydrogel has been developed for a variety of medical applications. Hydrogels have the property that they can be adjusted by a variety of chemical methods, so the design of the hydrogels provides a new way to deliver small size molecules, proteins, cells, and tissue regeneration [22]. Hydrogels are defined as water-swollen on hydrophlic polymer chains, which enable them to absorb large amounts of water and retained water constitutes at least $20 \%$ of its weight, that's why hydrogels are tunable properties as well as their versatile fabrication methods to be applied in biomedical applications, such as anti-acne patches [23].

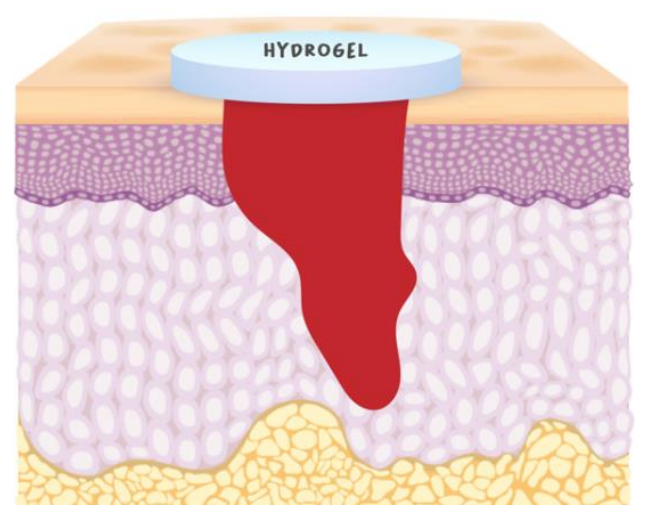

Fig. 3: Illustration of a hydrogel patches attached to a pimple
Some hydrogel patches usually contain triclosan, salicylic acid, or chlorhexidine diacetate as an anti-acne ingredient [22]. In a study by Lee et al. 2003, to make hydrogel patches, a polymer is usually used as a hydrogel base because of its hydrophilic and drug release properties. Polymer matrix used is Sodium polyacrylate (SPA) and carboxymethylcellulose (CMC, sodium salt). Besides that, there are also some additional substances such as Transcutol CG (TC, diethylene glycol monoethyl ether). TC is a potential transdermal permeation enhancer because it is non-toxic, biocompatible with the skin, and has excellent solubilizing performance. It is reported that it can also increase the skin accumulation of topical application compounds without simultaneously increasing in transdermal penetration. In order to obtain a semi-solid thin layer, in situ gelation technique is used; namely, the rate of the cross-linking reaction must be moderate and the compaction process cannot be completed before the fluid gel from the polymer is inserted into the film. Triclosan is a hydrophobic antibacterial agent that easily diffuses through the lipid skin layer. The in vitro antibacterial test results show that the patch can be effective not only on the skin underneath but on the surrounding skin as well. The addition of TC to this patch formulation has been shown to increase the accumulation of triclosan in the skin without a simultaneous increase in tarnsdermal permeation. This patch is also proven to adhere to facial skin well and can be detached from the skin without causing pain. So it can be concluded that the patch model in this study can be applied to the facial skin for acne treatment [24].

Hydrogels can be claasified based on their polymer composition [25]:

a. Homopolymeric hydrogels: A polymer network derived from a single types of monomer, which is the basic structural unit comprising of any polymer network. The homopolymers may have a cross-linked backbone structure, dependent on the nature of the monomer and the polymerization method.

b. Copolymeric hydrogels: These are consisted of two or more distinct monomer, including at least one hydrophilic component, assembled in random, block or alternate configurations along the chain of the polymer network 
c. Multipolymer: These, also known as interpenetrating polymeric hydrogel (IPN), are an important class of hydrogels made from two independent crosslinked and/or natural polymer components, confined in a network form. In a semi-IPN hydrogel, one component is a crosslinked polymer and the other component is a non-crosslinked polymer.

The advantages of hydrogels patches itself are (1) good biocompatibility, soft, and elastic on the skin (2) low toxicity potential (3) have good swelling behavior, especially on inflammation skin caused by acne and (4) the ability of drug protection against harsh environmental conditions. Meanwhile, the disadvantages are (1) not ideal for hydrophobic drugs (2) weak tensile of hydrogels caused an early release of the drug before give the effects at the target site [26]. Examples of formulations on hydrogel patches and their uses are described in table 6 .

\section{Hydrocolloid patches}

Hydrocolloid patches are is non medicated acne patch for treat acne. The function of this patch is to give skin hydration and improve the skin barrier. It can also used for enchancing skin smoothness and moisturizing effects. It can accelarate the barrier recovery because of acne. The example is glycerin as the main active ingredient. Glycerin can absorb and retaine water, can effectively relieve dry skin and heal acne faster [28]. Meanwhile, medicated acne patches is used to treat active acne, such as hydrogel patch that cointains triclsoan as antibacteria agent.

Table 6: Formulations on hydrogel patches and their uses

\begin{tabular}{ll}
\hline Formulation [24] & Function [27] \\
\hline Triclosan (TS) & Antibacterial agent \\
Transcutol GC (TC) & Solvent of TS \\
SPA and dihydroxyaluminum aminoacetate (DDA) & Gelling agent; emollient; emulsion stabilizer; stabilizing agent \\
Polyacrylic acid & Emulsifying agent; emulsion stabilizer; stabilizing agent \\
Tartaric acid & Acidyfing agent \\
Glycerin & Humectant; emollient; antimicrobial preservative; cosolvent \\
Carboxymethylcellulose (CMC, sodium salt) & Viscocity-increasing egnt; stabilizing agent \\
\hline
\end{tabular}

\section{Stability test}

According to the ICH guidelines, the accelerated stability test is used to perform a 6-month stability test on the transdermal patch under the following conditions: $40 \pm 2{ }^{\circ} \mathrm{C}$ temperature and $75 \pm 5 \%$ relative humidity (RH) to confirm the presence of the stability potential of the drug is the best-optimized formulation. Stability test was done by measuring concentration of active compound release after being stored for 6 mo using diffusion study, beside that, visual observation were also carried out. Visual observation includes shape, clarity, smoothness, homogeneity, viscovity, uniformity and flexibility.

Example of a stability test for the transdermal patch was done by Banerjee et al. (2014), from the results of these studies, it was found that the hydrogel transdermal patches had a fixed $\mathrm{pH}$ after being stored for 6 mo. The cumulative amounts of released drugs remained stable for 6 mo at 40C/75\% RH, and there was a small change in the complete drug release curve at $72 \mathrm{~h}$. Visual observation was also performed, and the visual characteristics of the optimized transdermal patch did not change during the analysis, especially in terms of shape, transparency, smoothness, uniformity, viscosity, uniformity, and flexibility. The ATR curve of the optimized transdermal patch remains stable during storage under accelerated pressure conditions [29].

\section{CONCLUSION}

The most commonly used patch to treat acne is the hydrogel patch, because it is waterproof on the surface to protect acne from secondary infection, it can absorb fluid on the pimples and make the pimples flatten, and the cost of manufacture is cheaper than Microneedle patches. The hydrogel patch can be stored stably for 6 mo at $40 \pm 2{ }^{\circ} \mathrm{C}$ and $75 \pm 5 \%$ relative humidity (RH). After 6 mo there is no change in $\mathrm{pH}$, the small change in the complete drug release profile at $72 \mathrm{~h}$, and the visual characteristics of the optimized transdermal patch during the analysis were not changed during the visual observation, especially in terms of shape, transparency, smoothness, uniformity, viscosity, uniformity and flexibility. In the pharmaceutical industry, polymers have been developed as the basis for hydrogel because of its hydrophilic and drug release properties.

\section{FUNDING}

Nil

\section{AUTHORS CONTRIBUTIONS}

All the authors contributed equally.

\section{CONFLICT OF INTERESTS}

Declared none

\section{REFERENCES}

1. NCBI. Acne vulgaris [internet]. Available from: https://www.ncbi.nlm.nih.gov/books/NBK459173. [Last accessed on 03 Jun 2021]

2. Zaenglein AL. Acne vulgaris. N Engl J Med. 2018;379(14):134352. doi: 10.1056/NEJMcp1702493, PMID 30281982.

3. Fox L, Csongradi C, Aucamp M, Du Plessis J, Gerber M. Treatment modalities for acne. Molecules. 2016;21(8):1-20. doi: 10.3390/molecules21081063, PMID 27529209.

4. Office of Women's Health (OASH). Acne [internet]. Gov/a-ztopics/acne. Available from: https://www.womenshealth. [Last accessed on 03 Jun 2021]

5. Zaenglein AL, Pathy AL, Schlosser BJ, Alikhan A, Baldwin HE, Berson DS, Bowe WP, Graber EM, Harper JC, Kang S, Keri JE, Leyden JJ, Reynolds RV, Silverberg NB, Stein Gold LF, Tollefson MM, Weiss JS, Dolan NC, Sagan AA, Stern M, Boyer KM, Bhushan R. Guidelines of care for the management of acne vulgaris. J Am Acad Dermatol. 2016;74(5):945-73.e33. doi: 10.1016/j.jaad.2015.12.037, PMID 26897386.

6. Eisenberg MJ, Afilalo J, Lawler PR, Abrahamowicz M, Richard H, Pilote L. Cancer risk related to low-dose ionizing radiation from cardiac imaging in patients after acute myocardial infarction. CMAJ. 2011;183(4):430-6. doi: 10.1503/cmaj.100463, PMID 21324846.

7. American Academy of Dermatologi. Association (AAD). Acne clinical guideline [internet]. Available from: https://www.aad.org/member/clinicalquality/guidelines/acne. [Last accessed on 03 Jun 2021]

8. Russell JJ. Topical therapy for acne. Am Fam Physician. 2000;61(2):357-66. PMID 10670502.

9. Titus S, Hodge J. Diagnosis and treatment of acne. Am Fam Physician. 2012;86(8):734-40. PMID 23062156.

10. Zhang Y, Feng P, Yu J, Yang J, Zhao J, Wang J, Shen Q, Gu Z. ROSresponsive microneedle patch for acne vulgaris treatment. Adv Therap. 2018;1(3). doi: 10.1002/adtp.201800035, PMID 1800035.

11. Li Y, Zhang H, Yang R, Laffitte Y, Schmill U, Hu W, Kaddoura M, Blondeel EJM, Cui B. Fabrication of sharp silicon hollow microneedles by deep-reactive ion etching towards minimally invasive diagnostics. Microsyst Nanoeng. 2019;5:41. doi: 10.1038/s41378-019-0077-y, PMID 31636931.

12. Pastore MN, Kalia YN, Horstmann M, Roberts MS. Transdermal patches: history, development and pharmacology. Br J Pharmacol. 2015;172(9):2179-209. doi: 10.1111/bph.13059, PMID 25560046.

13. Kapao N, Wattanutchariya W. Development of natural acne patch from local materials using quality function deployment technique. MATEC Web Conf. 2018;192:4-7. doi: 10.1051/matecconf/201819201050. 
14. Kuo CW, Chiu YF, Wu MH, Li MH, Wu CN, Chen WS, Huang CH. Gelatin/chitosan bilayer patches loaded with cortex Phellodendron amurense/centella asiatica extracts for antiacne application. Polymers. 2021;13(4):1-15. doi: 10.3390 /polym13040579, PMID 33671908.

15. Health line. From medicated to microneedle: 9 acne patches to try [internet]. Available from: https://www.healthline. communhealth/beauty-skin-care/acne-patches. [Last accessed on 03 Jun 2021].

16. Zhang T, Sun B, Guo J, Wang M, Cui H, Mao H, Wang B, Yan F. Active pharmaceutical ingredient poly(ionic liquid)-based microneedles for the treatment of skin acne infection. Acta Biomater. 2020;115:136-47. doi: 10.1016/j.actbio. 2020.08. 023, PMID 32853804.

17. Dharadhar S, Majumdar A, Dhoble S, Patravale V. Microneedles for transdermal drug delivery: a systematic review. Drug Dev Ind Pharm. 2019;45(2):188-201. doi: 10.1080/ 03639045.2018.1539497, PMID 30348022.

18. Tucak A, Sirbubalo M, Hindija L, Rahic O, Hadziabdic J, Muhamedagic K, Cekic A, Vranic E. Microneedles: characteristics, materials, production methods and commercial development. Micromachines. 2020;11(11):1-30. doi: 10.3390/mi11110961, PMID 33121041

19. Bariya SH, Gohel MC, Mehta TA, Sharma OP. Microneedles: an emerging transdermal drug delivery system. J Pharm Pharmacol. 2012;64(1):11-29. doi: 10.1111/j.2042-7158.2011. 01369.x, PMID 22150668.

20. Ita K. Ceramic microneedles and hollow microneedles for transdermal drug delivery: two decades of research. J Drug Deliv Sci Technol. 2018;44:314-22. doi: 10.1016/ j.jddst.2018.01.004.
21. Waghule T, Singhvi G, Dubey SK, Pandey MM, Gupta G, Singh M, Dua K. Microneedles: A smart approach and increasing potential for transdermal drug delivery system. Biomed Pharmacother. 2019;109:1249-58. doi: 10.1016/j.biopha. 2018.10.078, PMID 30551375.

22. Mandal A, Clegg JR, Anselmo AC, Mitragotri S. Hydrogels in the clinic. Bioeng Transl Med. 2020;5(2):e10158. doi 10.1002/btm2.10158, PMID 32440563.

23. Wang W, Narain R, Chapter ZH. 10-hydrogels. Polym Sci Nanotechnol. 2020;10:203-44

24. Lee TW, Kim JC, Hwang SJ. Hydrogel patches containing triclosan for acne treatment. Eur I Pharm Biopharm. 2003;56(3):407-12. doi: 10.1016/s0939-6411(03)00137-1, PMID 14602184

25. Garg S, Garg A, Vishwavidyalaya RD. Hydrogel: classification, properties, preparation and technical features. Asian J Biomaterial Res. 2016;2:163-70.

26. Narayanaswamy R, Torchilin VP. Hydrogels and their applications in targeted drug delivery. Molecules. 2019;24(3):603. doi: 10.3390/molecules24030603, PMID 30744011.

27. Rowe RC, Sheskey PJ. Handbook of pharmaceutical excipients. USA: Pharmaceutical Press; 2009.

28. De Paepe K, Wibaux A, Ward C, Rogiers V. Skin efficacy and biophysical assessment of glycerol-containing hydrocolloid patches. Skin Pharmacol Physiol. 2009;22(5):258-65. doi: 10.1159/000235553, PMID 19690451

29. Banerjee S, Chattopadhyay P, Ghosh A, Bhattacharya SS, Kundu A, Veer V. Accelerated stability testing of a transdermal patch composed of eserine and pralidoxime chloride for prophylaxis against ( \pm )-anatoxin A poisoning. J Food Drug Anal. 2014;22(2):264-70. doi: 10.1016/j.jfda.2014.01.022. 